\title{
Building a translational research program in Neurotology at University of Kansas Medical Center
}

\author{
Hinrich Staecker, David and Marilyn Zamierowski Professor, \\ Dept. of Otolaryngology, University of Kansas Medical Center \\ Kevin Sykes, Director of Clinical Research, Head and Neck Surgery, \\ University of Kansas Medical Center
}

$\mathrm{T}$ Translational research has been a common catchword in the NIH's effort to turn basic research into solutions for clinical problems. Despite a range of very successful programs in a range of medical specialties, the effort has not been largely successful for translational research of inner ear issues. Clinical otology currently suffers from a complete lack of medications to treat what really are amongst the most common neurodegenerative problems in man. Sensorineural hearing loss is almost ubiquitous by age 70 and vertigo represents one of the most common causes of primary care visits in the United States.

Compared to a similar organ system, the eye, there has been little true translational research despite years of basic science research and little understanding within our clinical subspecialty of how to actually make this happen. To most, translational research means disease research within an animal model. In this paper we will review our pathway to build a hearing and balance research infrastructure within the department of Otolaryngology Head and Neck Surgery at the University of Kansas Medical Center, and how we used that infrastructure to translate ongoing gene therapy research into human clinical trials.

The World Health Organization recently recognized the burden of hearing loss and estimates that $5 \%$ of the world's population is disabled by this condition (http://www.who.int/mediacentre/factsheets/fs300/en/). Currently we are limited to amplification (or in case of severe

hearing loss, cochlear implantation) as a means to restore hearing. Both of these are helpful but not ideal solutions to this very common problem. Development of medications for inner ear disease has been slow due to inability to biopsy the inner ear for analysis and the lack of correlation between clinical hearing and vestibular testing and site of lesion within the inner ear. Additionally it is only very recently that genetic testing has been available that can identify congenital or adult onset progressive hearing losses (Shearer, DeLuca et al. 2010).

Compared to other organ systems pathologic evaluation of human ear tissue is complex. What we know is derived from human temporal bones that have undergone decalcification and histologic evaluation often years after the active disease process is passed. The biology of the inner ear is complex and drugs that affect in ear function often will also affect the 
central nervous system, making systemically delivered medications difficult to apply. Basic science research has focused, broadly speaking, on several different research strategies with translational potential. These include delivery of growth factors to maintain our ganglion integrity, use of antioxidants are process factors to protect the interview trauma and hair cell regeneration.

Our lab has largely focused on the development of gene therapy technology for inner ear applications to help implement some discovery research going on in numerous other research groups. One of the relative advantages of the inner ear is that it can be locally accessed. Drugs can be delivered through the middle ear with diffusion of substances through the round window or through the stapediovestibular ligament or when larger molecules are involved, through direct injection into the inner ear. When we were initially looking for a translational research project to undertake, hair regeneration was an easy target since we have a quantifiable outcomes measure, namely the number of regenerated hair cells produced which could then be correlated to hearing or balance function. The molecular basis of hair cell regeneration has been established for a number of years. The transcription factor atonal/atoh1 is responsible for genesis of hair cells during development in vertebrates. Multiple researchers have demonstrated that the overexpression of atoh1 results in genesis of hair cells that are innervated and show recovery of function in mammalian models of ototoxicity(Baker, Brough et al. 2009). Delivery of atoh1 therefore could be a target for a translational research program.

When the project was undergoing its initial development phase we decided to target balance disorders caused by aminoglycoside ototoxicity. Patients who received gentamicin or other aminoglycosides for severe infections often end up with bilateral vestibular hypofunction despite adequate monitoring of blood aminoglycoside levels. Since we know the mechanism by which aminoglycosides damaged hair cells, a patient with loss of vestibular function with a history of aminoglycoside usage can be identified as a target for hair cell regeneration. An additional advantage of targeting the vestibular system is that there is no cochlear implant equivalent yet.

Despite early successes in regenerating hair cells in animal models of both hearing loss and vestibular dysfunction, numerous hurdles remained in the development process. Moving a drug candidate forward required calculation of dose response curves and understanding of the dosing relationships within the limited space of the inner ear. Additionally, analysis of potential downstream complications and longevity of regenerated hair cells had to be conducted. Animal hair cell loss models and drug delivery models had to be modified so that they could be used in contract research organizations where repeated experiments using GMP developed vector and GLP practices could be carried out. The recordkeeping and expense associated with this is generally outside of the ability of the basic research lab. Funding of these type of experiments is difficult, since it is not really discovery research and many 
study sections think that this type of research should be done by pharmaceutical companies. However, pharmaceutical companies will not pick up a potential product until this type of data is available to them at least in an early form.

In parallel to drug development efforts we had to establish a program for conducting clinical trials for inner ear disorders. Most clinical trial organizations or sections within the university hospital where clinical trials are conducted lack the ability to evaluate hearing and balance disorders. This is mainly due to the complex equipment and infrastructure needed to perform these types of evaluations. As always, putting new infrastructure in place requires funding. We set out to establish a hearing clinical trials program in 2007. Our initial efforts targeted device trials such as cochlear implants and implantable hearing aids. This provided the funding to cover the cost of an audiologist to perform testing and screen pa- tients. The establishment of the hearing clinical trial program was overseeing by Kevin Sykes (co-author). He had an appointment to the institutional review board (IRB) and initially oversaw integrity data collection and managed contacts. Our experience with running device trials and having infrastructure in place for hearing testing and a fulltime liaison to the research office allowed us to attract early-stage drug clinical trials for tinnitus and Ménière's disease. We then added a research fellow, generally a student who had completed college and wanted to spend a year in clinical research, to help manage the growing number of patients. In 2012 we hired a full-time research nurse which was needed to launch the gene therapy clinical trial. The growth in income from otology clinical trials during this time is shown in Figure 1.

In addition to covering the otology trials, putting clinical trial infrastructure into place has also opened opportunities

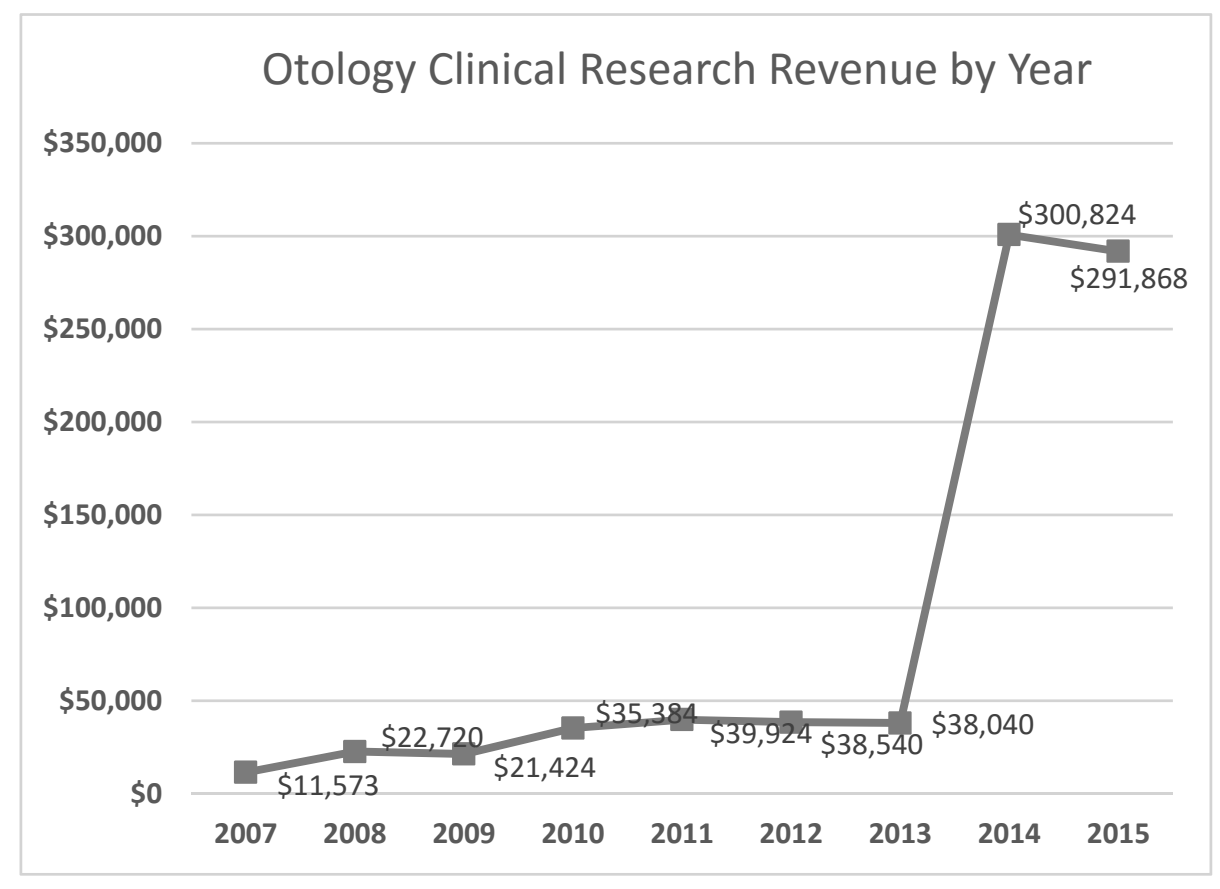

Figure 1: Income derived from the KU Otology clinical trial program over time. 
for other subspecialties within otolaryngology to provide clinical trials and bring in income to grow the academic mission. Establishing an infrastructure for complex trials such as inner ear gene therapy is vital, since these are high-risk trials that require time-consuming applications to the institutional IRB and prolonged contract negotiations with the trial sponsor. Additional staffing is also required to manage the flow of patients. Our current gene therapy trial requires two pretreatment visits and monthly visits for six months after delivery of the drug. Each of these visits takes two days, therefore scheduling and ensuring that audiological resources are available, especially when patients start to overlap, is important. All of this cannot be done by a lone clinician.

Having put a hearing research program together, we are now able to attract the interest of both preclinical and clinical stage research programs. The basic science lab has a subcontract with an early stage pharmaceutical company that is looking at our expertise in animal modeling to test a new nano particle for drug delivery to the middle ear space. The clinical research unit is now involved with two new phase 3 trials for tinnitus and for Ménière's disease. It is only due to our publication record and our track record of working with several contract research organization and being able to provide clinical material for a number of trials that we are able to do this. Academic institutions generally cannot compete with private institutions that make money from clinical trials.

Most of the private providers of these services focus on straightforward internal medicine type clinical trials and are competitive because of lower overhead costs and more straightforward contract negotiations with the sponsor. Many of these institutions also use private IRBs which are often more straightforward to navigate than a University IRB. The advantage of being an academic center is that we have a large base of patients with a number of rare diseases, and have infrastructure to support the testing and treatment of these diseases. This is true not only of neurotology but other subspecialties as well. Establishment of a successful translational research program requires a track record and most of all, an experienced and dedicated support structure.

\section{References}

Baker, K., D. E. Brough and H. Staecker (2009). "Repair of the vestibular system via adenovector delivery of Atoh1: a potential treatment for balance disorders." Adv Otorhinolaryngol 66: 52 63.

Shearer, A. E., A. P. DeLuca, M. S. Hildebrand, K. R. Taylor, J. Gurrola, 2nd, S. Scherer, T. E. Scheetz and R. J. Smith (2010). "Comprehensive genetic testing for hereditary hearing loss using massively parallel sequencing." Proc Natl Acad Sci U S A 107(49): 21104-21109. 